\title{
Maximum induced forests in random graphs
}

\author{
M. Krivoshapko*, M. Zhukovskii雨
}

\begin{abstract}
We prove that with high probability maximum sizes of induced forests in dense binomial random graphs are concentrated in two consecutive values.
\end{abstract}

\section{Introduction}

Given a graph $G$, its subgraph $H$ is called induced, if any two vertices $u, v$ of $H$ are adjacent in $H$ if and only if they are adjacent in $G$. The independence number of $G$ is the maximum number of vertices in an induced subgraph of $G$ that contains no edges. A forest is an acyclic graph. Everywhere below, the size of $G$ is the number of vertices in $G$.

It is very well known (see [1, 4, 8, 9]) that the independence number of the binomial random graph $G(n, p=$ const) (in this graph, every pair of distinct vertices from $\{1, \ldots, n\}$ is adjacent with probability $p$ independently of the others) is concentrated in two consecutive values. In other words, there is a function $f(n)$ such that with high probability (i.e., with probability tending to 1 as $n \rightarrow \infty$ ) the independence number of $G(n, p)$ equals either $f(n)$ or $f(n)+1$. In [3], the same concentration result was obtained for the maximum size of an induced path in $G(n, p)$ and for the maximum size of an induced cycle in $G(n, p)$. Finally, in [5], the following 2-point concentration result was obtained for the maximum size of an induced tree.

Theorem $1([5])$. There exists an $\varepsilon>0$ such that with high probability the maximum size of an induced tree in $G(n, p)$ equals either $\left\lfloor 2 \log _{1 /(1-p)}(e n p)+2+\varepsilon\right\rfloor$ or $\left\lfloor 2 \log _{1 /(1-p)}(e n p)+3+\varepsilon\right\rfloor$.

In this paper, we prove that the same holds true for the maximum size of an induced forest in $G(n, p)$.

\footnotetext{
${ }^{*}$ Moscow Institute of Physics and Technology (National Research University), Department of Discrete Mathematics, Dolgoprudny, Moscow Region, Russian Federation.

${ }^{\dagger}$ Adyghe State University, Caucasus mathematical center, Maykop, Republic of Adygea, Russian Federation; The Russian Presidential Academy of National Economy and Public Administration, Moscow, Russian Federation; Moscow Center for Fundamental and Applied Mathematics, Moscow, Russian Federation.

zhukmax@gmail.com
} 


\section{The result}

Theorem 2. There exists an $\varepsilon>0$ such that with high probability the maximum size of an induced forest in $G(n, p)$ equals either $\left\lfloor 2 \log _{1 /(1-p)}(e n p)+2+\varepsilon\right\rfloor$ or $\left\lfloor 2 \log _{1 /(1-p)}(e n p)+3+\varepsilon\right\rfloor$.

Let $\varepsilon>0$ be the constant from Theorem 1. Let $T_{n}, F_{n}$ be the maximum sizes of an induced tree and an induced forest in $G(n, p)$ respectively. Since a tree is a forest itself, we get that $F_{n} \geq T_{n}$. Then, the lower bound in Theorem 2 follows from Theorem 1. The upper bound of Theorem 1 follows from Markov's inequality. More formally, let $X_{n}$ be the number of induced trees in $G(n, p)$ of size $\left\lfloor 2 \log _{1 /(1-p)}(e n p)+4+\varepsilon\right\rfloor$. In [5], it is proven that $\mathrm{E} X_{n} \rightarrow 0$. By Markov's inequality, it immediately implies that $\mathrm{P}\left(X_{n}=0\right) \rightarrow 1$. Now, let $Y_{n}$ be the number of induced forests in $G(n, p)$ of size $\left\lfloor 2 \log _{1 /(1-p)}(e n p)+4+\varepsilon\right\rfloor$. In Section [3, we prove the following lemma.

Lemma 3. There exists $C>0$ such that $\mathrm{E} Y_{n} \leq C \mathrm{E} X_{n}$.

Lemma 3 and the above arguments imply that $\mathrm{P}\left(Y_{n}>0\right) \leq \mathrm{E} Y_{n} \rightarrow 0$ as $n \rightarrow \infty$. Theorem 2 follows.

\section{Proof of Lemma 3}

Set $K=\left\lfloor 2 \log _{1 /(1-p)}(e n p)+4+\varepsilon\right\rfloor$. For $\ell \in\{1, \ldots, K\}$, let $Y_{n, \ell}$ be the number of induced forests in $G(n, p)$ on $K$ vertices with $\ell$ connected components. For $k \geq 2$ and $\ell \in\{1, \ldots, k\}$, let $\varphi_{\ell}(k)$ be the number of forests with $\ell$ components on a labeled set of $k$ vertices. Then

$$
\begin{gathered}
\mathrm{E} Y_{n}=\sum_{\ell=1}^{K} \mathrm{E} Y_{n, \ell}=\sum_{\ell=1}^{K}\left(\begin{array}{l}
n \\
K
\end{array}\right) \varphi_{\ell}(K) p^{K-l}(1-p)^{\left(\begin{array}{c}
K \\
2
\end{array}\right)-K+l}= \\
\left(\begin{array}{l}
n \\
K
\end{array}\right) p^{K-1}(1-p)^{\left(\begin{array}{c}
K \\
2
\end{array}\right)-K+1} \sum_{\ell=1}^{K} \varphi_{\ell}(K) p^{-\ell+1}(1-p)^{\ell-1}=\mathrm{E} X_{n} \sum_{\ell=1}^{K} g_{\ell}(K),
\end{gathered}
$$

where

$$
g_{\ell}(k)=\frac{\varphi_{\ell}(k) \cdot((1-p) / p)^{\ell-1}}{k^{k-2}} .
$$

Set $g_{\ell}(k)=0$ for all $\ell>k$. 
Since (see [10, Section 4.3]) $\lim _{k \rightarrow+\infty} \frac{\varphi_{\ell}(k)}{k^{k-2}}=\frac{(1 / 2)^{\ell-1}}{(\ell-1) !}$, we get that $\lim _{k \rightarrow \infty} g_{\ell}(k)=$ $\frac{\left([(1-p) /(2 p)]^{\ell-1}\right.}{(\ell-1) !}$. Then, due to Tannery's theorem [6, Theorem 3.30], to prove that

$$
\lim _{k \rightarrow \infty} \sum_{\ell=1}^{k} g_{\ell}(k)=\sum_{\ell=1}^{\infty} \lim _{k \rightarrow \infty} g_{\ell}(k)=\sum_{\ell=1}^{\infty} \frac{\left([(1-p) /(2 p)]^{\ell-1}\right.}{(\ell-1) !}<\infty
$$

it is sufficient to show that, for every $\ell \in \mathbb{N}$, there exists $M_{\ell}$ such that, for all $k \geq 2$, $g_{\ell}(k)<M_{\ell}$ and $\sum_{\ell=1}^{\infty} M_{\ell}<\infty$. Notice that, from (11) and (2), Lemma 3 follows.

Let $\ell \geq 2, m \in\{1, \ldots, k-1\}$. Since the number of forests on $\{1, \ldots, k\}$ with $\ell$ components such that the component containing the vertex $k$ has exactly $k-m$ vertices equals $\left(\begin{array}{c}k-1 \\ m\end{array}\right)(k-m)^{k-m-2} \varphi_{\ell-1}(m)$, we get the following (recall that $\varphi_{\ell-1}(m)=0$ when $m<\ell-1)$ :

$$
\varphi_{\ell}(k)=\sum_{m=\ell-1}^{k-1}\left(\begin{array}{c}
k-1 \\
m
\end{array}\right)(k-m)^{k-m-2} \varphi_{\ell-1}(m) .
$$

Therefore,

$$
g_{\ell}(k)=\frac{1-p}{p} \sum_{m=\ell-1}^{k-1}\left(\begin{array}{c}
k-1 \\
m
\end{array}\right) \frac{m^{m-2}(k-m)^{k-m-2}}{k^{k-2}} g_{\ell-1}(m) .
$$

Let $M_{\ell}=\max _{k \in \mathbb{N}} g_{\ell}(k)$ (the maximum exists since $g_{\ell}(k)$ has a finite limit as $k \rightarrow \infty)$. Then, (3) implies that

$$
g_{\ell}(k) \leq \frac{1-p}{p} M_{\ell-1} \sum_{m=\ell-1}^{k-1}\left(\begin{array}{c}
k-1 \\
m
\end{array}\right) \frac{m^{m-2}(k-m)^{k-m-2}}{k^{k-2}}=\frac{1-p}{p} M_{\ell-1} \sum_{m=\ell-1}^{k-1} f(m, k),
$$

where

$$
f(m, k)=\left(\begin{array}{c}
k-1 \\
m
\end{array}\right) \frac{m^{m-2}(k-m)^{k-m-2}}{k^{k-2}} .
$$

Let us show that there exists $C>0$ such that $\sum_{m=\ell-1}^{k-1} f(m, k) \leq \frac{C}{\ell}$ for all $k$ and $\ell$. If the latter is true, then, by (4), we get that $M_{\ell} \leq \frac{1-p}{p} \frac{C}{\ell} M_{\ell-1}$ that immediately implies the desired finiteness of $\sum_{\ell=1}^{\infty} M_{\ell}$.

By Stirling's approximation $\sqrt{2 \pi n}(n / e)^{n}<n !<\sqrt{2 \pi n}(n / e)^{n} e^{\frac{1}{12} n}([12])$, we get that, for all $k \geq 3$ and $m \leq k-2$,

$$
f(m, k)<\frac{\sqrt{2 \pi(k-1)}(k-1)^{k-1} e^{\frac{1}{12(k-1)}}}{2 \pi \sqrt{m(k-1-m)} m^{m}(k-1-m)^{k-1-m}} m^{m-2} \frac{(k-m)^{k-m-2}}{k^{k-2}}=
$$




$$
\begin{gathered}
\sqrt{\frac{(k-1)}{2 \pi(k-1-m)}} \frac{k^{2}}{(k-m)(k-1)}\left(1-\frac{1}{k}\right)^{k}\left(1+\frac{1}{k-m-1}\right)^{k-m-1} \frac{e^{\frac{1}{12(k-1)}}}{m^{2} \sqrt{m}}< \\
c \frac{k^{3 / 2}}{m^{5 / 2}(k-m)^{3 / 2}}
\end{gathered}
$$

for some constant $c>0$. If $m=k-1$, then $f(m, k)=\frac{(k-1)^{k-3}}{k^{k-2}}<\frac{1}{k}$. So, the above bound is also true in this case.

Notice that the function $v(x)=\frac{k^{3 / 2}}{x^{5 / 2}(k-x)^{3 / 2}}$ is convex on $(0, k)$. Therefore, for all $k \geq 2$ and $2 \leq \ell \leq k$,

$$
\begin{aligned}
& \sum_{m=\ell-1}^{k-1} \frac{k^{3 / 2}}{m^{5 / 2}(k-m)^{3 / 2}} \leq v(k-1)+v(\ell-1)+\int_{\ell-1}^{k-1} v(x) d x= \\
& \frac{k^{3 / 2}}{(k-1)^{5 / 2}}+\frac{k^{3 / 2}}{(\ell-1)^{5 / 2}(k-\ell+1)^{3 / 2}}-\left.\frac{2\left(k^{2}+4 k x-8 x^{2}\right)}{3 k^{3 / 2} x^{3 / 2} \sqrt{k-x}}\right|_{\ell-1} ^{k-1}< \\
& \frac{6}{k}+\frac{3}{\ell-1}+\frac{2\left(3 k^{2}-12 k+8\right)}{3 k^{3 / 2}(k-1)^{3 / 2}}+\frac{2\left(k^{2}+4 k(\ell-1)-8(\ell-1)^{2}\right)}{3 k^{3 / 2}(\ell-1)^{3 / 2} \sqrt{k-\ell+1}} .
\end{aligned}
$$

Since

$$
\frac{2\left(3 k^{2}-12 k+8\right)}{3 k^{3 / 2}(k-1)^{3 / 2}}<\frac{6(k-2)^{2}}{3 k^{3 / 2}(k-1)^{3 / 2}}<\frac{2 \sqrt{k-1}}{3 k \sqrt{k}}<\frac{2}{3 k}
$$

and

$$
\begin{gathered}
\frac{2\left(k^{2}+4 k(\ell-1)-8(\ell-1)^{2}\right)}{3 k^{3 / 2}(\ell-1)^{3 / 2} \sqrt{k-\ell+1}}<\frac{2\left[(k+2(\ell-1))^{2}-16(\ell-1)^{2}\right]}{3 k^{3 / 2}(\ell-1) \sqrt{(k-(\ell-1))(\ell-1)}}< \\
\frac{2(k-2(\ell-1))(k+6(\ell-1))}{k^{2}(\ell-1)}<\frac{2(k+6 k)}{k(\ell-1)}=\frac{14}{\ell-1},
\end{gathered}
$$

the desired bound follows.

\section{Discussions}

We have proved that with high probability the maximum size of an induced forest in $G(n, p)$ is concentrated in 2 consecutive points: $\left\lfloor 2 \log _{1 /(1-p)}(e n p)+2+\varepsilon\right\rfloor$ and $\left\lfloor 2 \log _{1 /(1-p)}(e n p)+3+\varepsilon\right\rfloor$. Moreover, from our arguments, it follows that the difference between it and the maximum size of an induced tree is not bigger than 1 . It would be interesting to extend these results for $p=o(1)$. In sparse settings (particularly, for $p=c / n$ and $p=c \ln n / n$ ), some bounds are known [2, 7, 11] for the maximum size of an induced tree, but even the exact asymptotics of these maximum sizes are unknown. 


\section{Acknowledgements}

The reported study was funded by RFBR according to the research project $\mathrm{N} 20$ 04-60524.

\section{References}

[1] B. Bollobás, P. Erdős, Cliques in random graphs, Math. Proc. Camb. Phil. Soc. 80 (1976) p. 419-427.

[2] W. Fernandez de la Vega, The largest induced tree in a sparse random graph, Random Struct. Alg. 9:1-2 (1996) p. 93-97.

[3] K. Dutta, C.R. Subramanian, On Induced Paths, Holes and Trees in Random Graphs Proc. ANAL-CO (2018). p. 168-177.

[4] G. R. Grimmett, C. J. H. McDiarmid, On colouring random graphs, Math. Proc. Cambridge Philos. Soc. 77 (1975) p. 313-324.

[5] D. Kamaldinov, A. Skorkin, M. Zhukovskii, Maximum sparse induced subgraphs of the binomial random graph with given number of edges, Discrete mathematics 34:2 (2021), 112205.

[6] P. Loya, Amazing and Aesthetic Aspects of Analysis, Springer, 2017.

[7] T. Łuczak, Z. Palka, Maximal induced trees in sparse random graphs, Discrete Mathematics, 72:1-3 (1988) pp. 257-265.

[8] D. W. Matula, The Employee Party Problem, Notices of the American Mathematical Society, 19:2 (1972) p. A-382.

[9] D. W. Matula, The largest clique size in a random graph, Tech. Rep. Dept. Comp. Sci., Southern Methodist University, Dallas, Texas, 1976.

[10] J. W. Moon, Counting Labelled Trees, Canadian Mathematical Congress, 1970.

[11] Z. Palka and A. Ruciński, On the order of the largest induced tree in a random graph, Discrete Applied Mathematics, 15:1 (1986) pp. 75-83.

[12] H. Robbins, A Remark on Stirling's Formula, The American Mathematical Monthly, 62:1 (1955), p. 26-29. 